This item was submitted to Loughborough's Research Repository by the author.

Items in Figshare are protected by copyright, with all rights reserved, unless otherwise indicated.

Effective visualisation of design versions : visual storytelling for design reuse

PLEASE CITE THE PUBLISHED VERSION

PUBLISHER

(C) Springer Verlag

VERSION

AM (Accepted Manuscript)

LICENCE

CC BY-NC-ND 4.0

REPOSITORY RECORD

Demian, Peter, and Renate Fruchter. 2019. "Effective Visualisation of Design Versions : Visual Storytelling for Design Reuse". figshare. https://hdl.handle.net/2134/4090. 
This item was submitted to Loughborough's Institutional Repository (https://dspace.lboro.ac.uk/) by the author and is made available under the following Creative Commons Licence conditions.

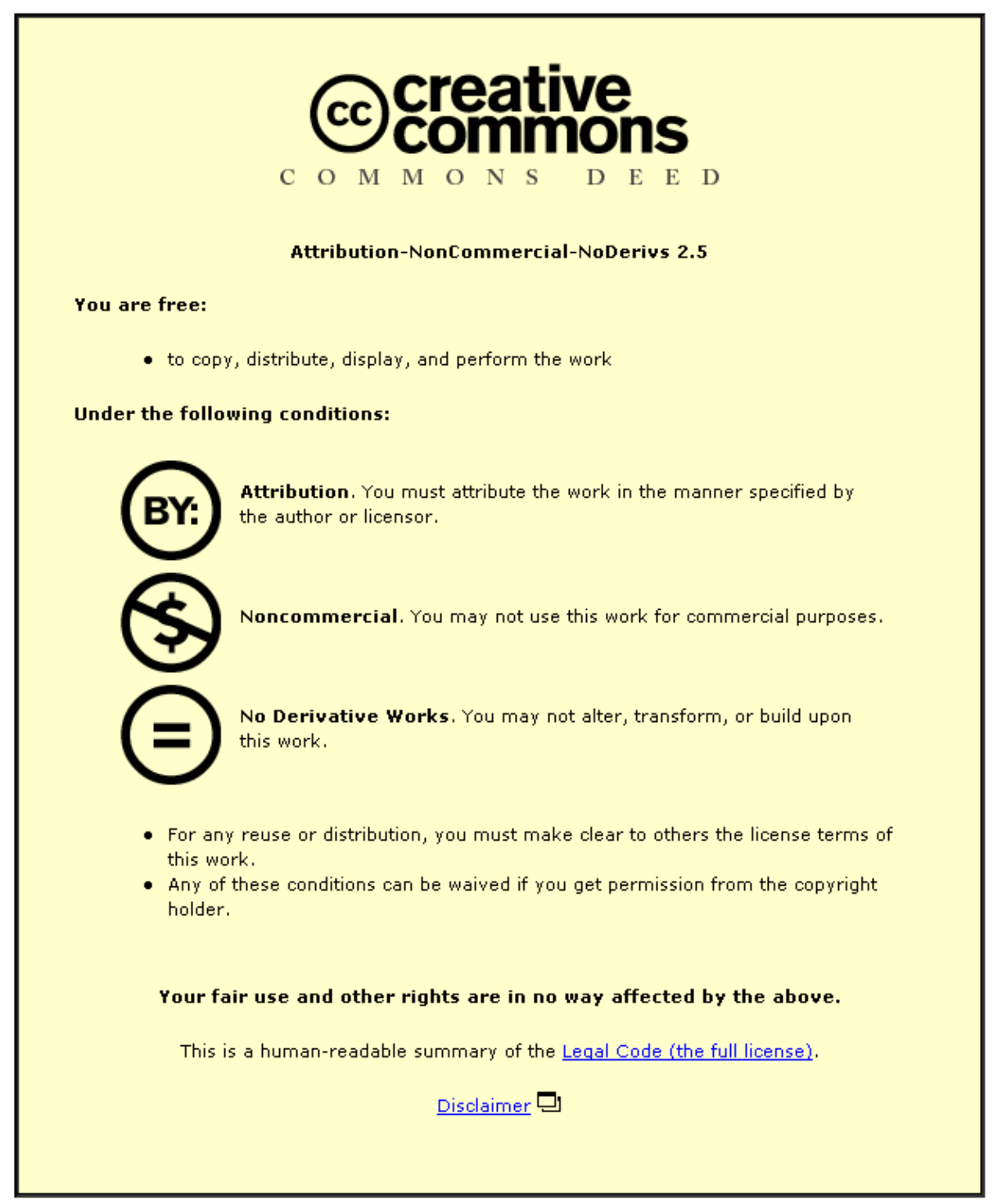

For the full text of this licence, please go to: http://creativecommons.org/licenses/by-nc-nd/2.5/ 
Demian, P. and Fruchter, R., 2009, "Effective visualisation of design versions: visual storytelling for design reuse" Research in Engineering Design, 19(4) 193-204.

\title{
Effective Visualisation of Design Versions: Visual Storytelling for Design Reuse
}

\author{
PETER DEMIAN $^{1}$ AND RENATE FRUCHTER ${ }^{2}$ \\ 1. Lecturer, Department of Civil and Building Engineering, Loughborough \\ University, LE11 3TU,UK, P.Demian@lboro.ac.uk \\ 2. Director of Project Based Learning Lab, Department of Civil and \\ Environmental Engineering, Stanford University, Stanford, CA 94305, USA, \\ fruchter@stanford.edu
}

\section{Abstract}

Improving and supporting the process of design knowledge reuse can increase productivity, improve the quality of designs and lead to corporate competitive advantage. Whereas internal knowledge reuse (reusing knowledge from one's personal memory or experiences) is very effective, external knowledge reuse (reusing knowledge from an external digital or paper archive) often fails. This paper studies the value of the storytelling paradigm in supporting reuse from an external repository. Based on a formalisation of the internal reuse process from ethnographic studies, a prototype system, CoMem (Corporate Memory) is presented, which supports the reuse process, specifically the steps of finding and understanding reusable items. This paper focuses on the ability of designers to understand designs that are found in corporate repositories. It is argued that in order to understand and reuse a found design, the designer needs to see the evolution of that design during the original design process. An Evolution History Explorer module of the CoMem system is presented that uses a storytelling metaphor and lays out versions visually side-by-side. A formal user evaluation of CoMem supports the hypotheses that (1) exploring the evolution of a design improves the reuse process, and (2) that visual storytelling is an effective paradigm for supporting that exploration.

\section{Introduction}

The average designer, whether consciously or subconsciously, draws from a vast well of previous design experience when solving new design problems. "All design is redesign" (Leifer 1997). The reused content can include experience acquired by the individual or by his/her mentors or professional community. This activity of recalling from past design experiences is referred to as design knowledge reuse. The term "design knowledge reuse" (rather than simply "design reuse") is used to indicate that what is reused is not limited to the product, or previously designed artefacts, but also includes the process, i.e., the knowledge and expertise ingrained in hose previous designs.

This paper looks into the importance of exploring the evolution of a design before reusing that design. How can the evolution of a design be visualized in order to offer the maximum possible support for the understanding and effective reuse of previous designs?

This research distinguishes between two types of reuse:

- Internal knowledge reuse: a designer reusing knowledge from his/her own personal experiences (internal memory).

- External knowledge reuse: a designer reusing knowledge from an external knowledge repository (external memory).

Internal knowledge reuse is an effective process, which researchers place at the very centre of human intelligence (Schank 1990). Human learning, generalization 
Demian, P. and Fruchter, R., 2009, "Effective visualisation of design versions: visual storytelling for design reuse" Research in Engineering Design, 19(4) 193-204.

and prediction all depend on the mind's ability to remember past events. On the other hand external knowledge reuse often fails, for example because knowledge is not externally archived or captured, it is captured out of context, or there are no mechanisms or software tools to facilitate reuse of captured content.

Empirical observations of designers at work (Demian and Fruchter 2006) show that internal knowledge reuse is effective because:

- The designer can quickly find (mentally) reusable items.

- The designer can remember the context of each item, and can therefore understand it and reuse it more effectively.

When studying the way experienced designers mentor novices, and the way experts describe previous designs to novices, it appears that there are two important contextual dimensions that are explored and which lead to understanding of the design of the found item (and therefore effective reuse of the item): the project context, i.e. related items in the same designed artefact or building project, and the evolution history, i.e. the evolution of the item in question and the rationale driving this design evolution. The three key activities in the design knowledge reuse process are:

- Finding a reusable item.

- Exploring this item's project context that leads to understanding of the design of the found item.

- Exploring this item's evolution history that leads to understanding of the design of the found item.

This paper focuses on evolution history exploration. The evolution history is the record of the iterations a design progresses through, from an abstract idea or a set of requirements to a fully designed physical entity. Given an item from a previous project, three directions of exploration were identified from the ethnographic study:

- BACKWARDS: From detailed to conceptual. Designers move backwards to trace the concepts that were explored early on in the design of an item. In the ethnographic study, designers were observed to retrieve and explore early sketches depicting undeveloped design concepts.

- FORWARDS: From conceptual to detailed. Designers move forwards to follow the evolution of this item into a fully designed physical component. In the ethnographic study, designers reusing a past design were observed to explore that design's evolution into a fully detailed component in a CAD file, and even retrieve photographs of the design as built.

- SIDEWAYS: From alternative to alternative. Designers also move sideways to explore the different alternatives that were considered at any stage in the design process. Perhaps an alternative that was abandoned for the original project could now be reused.

These observations of internal knowledge reuse are used as the basis for improving external knowledge reuse. The general hypothesis of this research is that if the designer's interaction with the repository enables him/her to:

- Rapidly find relevant items of design knowledge,

- View each item in context in order to understand it, specifically:

o Explore its project context

o Explore its evolution history 
Demian, P. and Fruchter, R., 2009, "Effective visualisation of design versions: visual storytelling for design reuse" Research in Engineering Design, 19(4) 193-204.

then the process of reuse will be improved. This improved reuse will lead to higher quality design solutions, and save time and money.

\section{Points of Departure}

This research was part of a series of research projects on design knowledge management conducted at the Project-Based Learning Lab at Stanford University. These projects are based on Schön's reflective practitioner paradigm of design (Schön 1983). Schön argues that every design task is unique, and that the basic problem for designers is to determine how to approach such a single unique task. Schön's reflective practitioner paradigm forms the conceptual basis of knowledge capture in the Semantic Modeling Engine (SME) (Fruchter 1996), which enables designers to map objects from a shared CAD product model to multiple semantic representations.

The ProMem (Project Memory) system (Fruchter et al. 1998) takes SME as its point of departure and adds to it the time dimension. ProMem captures the evolution of the project at the three levels of granularity identified by SME to emulate the structure of project knowledge: project, discipline, and component. ProMem automatically versions each SME object every time a change is made in the design or additional knowledge is created and added to these items. ProMem maintains this version history without imposing additional work on the original designers to document their design process. Instead, ProMem transparently captures design evolution as the team develops the CAD model by allowing individual team-members to exchange textual massages to broadcast or negotiate changes.

This paper presents CoMem (Corporate Memory), a system that is built on top of ProMem. CoMem extends ProMem firstly by grouping the accumulated set of project memories into a corporate memory, and secondly by supporting the designer in reusing design content from this corporate memory in new design projects. The content offered for reuse by CoMem is in the SME format: CAD models annotated with hierarchical semantic objects, multiple projects, each project containing multiple disciplines (or subsystems), each discipline consisting of multiple component objects. In addition, a version history is maintained for each project, discipline, or component object.

\section{Related Research}

Beyond CoMem's research predecessors (SME and ProMem), several other research studies focus specifically on reuse. Research studies on design knowledge reuse focus either on the cognitive aspects or on the computational aspects. Research into the cognitive aspects of reuse has helped to identify the information needed by designers. Kuffner and Ullman (1990) found that the majority of information requested by mechanical engineers was concerning the operation or purpose of a design object, information that is not typically captured in standard design documents (drawings and specifications). Finger (1998) observed that designers rarely use CAD tools to help them organize and retrieve design information.

On the computational side, research into design knowledge reuse focuses on knowledge representation and reasoning. Knowledge representation ranges from 
Demian, P. and Fruchter, R., 2009, "Effective visualisation of design versions: visual storytelling for design reuse" Research in Engineering Design, 19(4) 193-204.

informal classification systems for standard components (for example Culley 1998) to more structured design rationale approaches (Regli et al. 2000 gives an overview). Highly structured representations of design knowledge can be used for reasoning. Two common approaches are case-based reasoning (CBR)and modelbased reasoning. AskJef (Barber et al. 1992) is a noteworthy CBR system which supports interface designers by providing design examples, principles, guidelines, errors and stories. This research contrasts with many CBR efforts in that it does not attempt to automate the processes of case retrieval and case adaptation, but instead attempts to provide the maximum possible support to the human designer performing these tasks. This approach of supporting rather than replacing human designers is becoming increasingly common in CBR research (Simoff and Maher 1998, Popova et al. 2002, Aha et al. 2006). CBR has also been applied to many educational systems (Kolodner et al. 2006).

In the field of software design, code reuse is an active research topic (for example: Zieliński et al. 1995, Kazman and Carrière 1998, Jerding and Stasko 1994, Ye and Fischer 2002). All these projects emphasize the importance of the understanding of archived components. Retkowsky (1998) lists the steps for software reuse as finding, understanding, adapting, and integrating.

Product Data Management (PDM) is a field of research on computational aspects of reuse that does not necessarily aspire to design reasoning. PDM systems sometimes include versioning functionality (Harder et al. 2000) and are increasingly web-based (Liu and Xu 2001).

PDM systems are often compared to Software Configuration Management (SCM) systems used for software design (Association of Swedish Engineering Industries 2001, Crnkovic et al. 2001, El-khoury 2005). PDM is often integrated with workflow management (McClatchey, et al. 1998) which helps to give rise to versioning functionality. Some research efforts focus specifically on versioning (e.g. Schonhoff et al. 2001) but no research was encountered which expressly concentrates on visualising version histories to tell a story.

State of the art PDM systems such as Teamcenter by Siemens aim to provide a single source of all content related to a product. This resource can remain active over the product's entire lifecycle. Windchill by PTC, Enovia by 3DS, and MatrixOne are all similar but focus on very slightly different aspects such as process management, team collaboration, or electronic commence. Unlike CoMem, PDM systems do not specifically target the archiving of content after a project is complete and the reuse of that content in later projects.

Finally, many related research efforts recognize the value of informal design evolution content in understanding design rationale and effectively reusing previously designed artefacts (Carroll et al. 1994, Malmqvist 1995, Ball et al. 2001).

\section{The CoMem Design Reuse System}

CoMem (Fruchter and Demian 2002) is based on the principle of "overview first, zoom and filter, and then details-on-demand" (Shneiderman 1999). Based on the three reuse activities identified- find, explore project context, explore evolution 
Demian, P. and Fruchter, R., 2009, "Effective visualisation of design versions: visual storytelling for design reuse" Research in Engineering Design, 19(4) 193-204.

history - CoMem has three corresponding modules: an Overview, a Project Context Explorer, and an Evolution History Explorer (Figure 1).

\begin{tabular}{|c|c|c|}
\hline Reuse step & & User interaction \\
\hline Find reusable item & \multirow{3}{*}{$\begin{array}{c}\text { "overview first, zoom and } \\
\text { filter, and then details-on- } \\
\text { demand" }\end{array}$} & Overview \\
\hline Explore item's & & Evolution History Explorer \\
\hline Explore item's project context & & Project Context Explorer \\
\hline
\end{tabular}

Figure 1: Transformation from observed reuse steps to CoMem user interactions.

The Overview supports the designer in finding reusable items by representing projects, disciplines, and components as nested rectangles using the squarified treemap visualization (Bruls et al. 1999). Each rectangle is coloured according to its relevance to the designer's current design task. The metaphor is that of a geographical map, giving the designer an indication of which "regions" of the corporate memory contain potentially reusable items.

Once the user has selected an item from the Overview, the Project Context Explorer supports the designer in exploring this item's project context. This module uses a fisheye lens metaphor and balances local detail with global context. It shows the project and subsystem to which this item belongs, as well as related components and disciplines that would help the designer understand the found item.

In the third module, the Evolution History Explorer, the designer can explore the evolution history of any item selected from the Overview. Using a visual storytelling metaphor, this view tells the story of how this item evolved from an abstract idea to a fully designed physical artefact.

\section{The Importance of Exploring the Evolution of Designs}

The Evolution History Explorer should enable the designer to explore the evolution history of a given project, discipline, or component object over time. The exploration can be backwards (exploring early concepts), forwards (exploring detailed designs later in the design process), or sideways (exploring design alternatives). This is important for two reasons:

- Reusing intermediate versions. The reusable content may be at an intermediate stage of the evolution of the item. For example, a fully designed CAD model of a cooling tower frame may not be useful, whereas an early sketch showing the load path concept is. Perhaps an early design alternative that was abandoned for the original project can now be reused.

- Understanding a particular version and gaining design expertise. Even if the final design can potentially be reused, the evolution of this design needs to be studied in order to understand this item and make an informed decision about whether and how to reuse it. In some cases the design process is more important than the product. Importing a CAD component from a previous project will bring about an immediate improvement in productivity. However this can be small compared to the lasting improvement in productivity which results from understanding the design rationale and, as a result, gaining valuable design expertise to be applied to future projects. 
Demian, P. and Fruchter, R., 2009, "Effective visualisation of design versions: visual storytelling for design reuse" Research in Engineering Design, 19(4) 193-204.

These two objectives serve as yardsticks against which any solution for supporting evolution history exploration can be assessed. The extent to which these objectives are met depends not only on the user interface but also on the nature of the evolution history data available.

We model the evolution history for a CoMem object as a tree structure. Each time the system detects a change in the design, a new version (node) of the object is created and linked to its parent. Each version is linked to specific graphic objects from the CAD model. In addition, a version can also have notes and data objects, which are attached to the product model in the same way that designers in current practice annotate paper drawings with handwritten notes. Notification objects are used to solicit feedback, give approval, broadcast changes, or initiate negotiations in the same way that designers interact during the design process. Hyperlink objects are used to share documents with team members by linking the documents to the specific component or discipline objects in the shared 3D building model.

Collectively, the content attached to these versions describes both the nature of the evolution, i.e., how the design evolved, as well as the rationale for this evolution, i.e. why it evolved the way it did. This paper looks into how such a version history can be visualized and how the user can interact with it in order to offer the maximum possible support for the above objectives, especially understanding a particular version and gaining design expertise, which is more challenging.

\section{The Storytelling Metaphor}

CoMem uses a storytelling metaphor for the Evolution History Explorer. In its most literal meaning, a story is simply a narrative of events. However stories have additional expressive content, weaving details, characters, and events into a whole that is greater than the sum of its parts (Simmons 2001). In a traditional Jewish allegory (recounted in Simmons 2001) Truth is turned away from every door in the village because her nakedness frightens the people. When Parable finds her huddled in a corner, she has pity on her and takes her home. There, Parable dresses Truth in story and sends her out again. Clothed in story, Truth once again knocks on the villagers' doors, and this time is readily welcomed into their houses.

The use of stories has been studied in many contexts, including influencing people in business settings (Simmons 2001), bringing about social change (Davis 2002), as a literary art form (Fulford 1999) and as graphic art form (Eisner 1996). The educational value of stories is particularly recognised. Ferguson et al. (1992) define an Aesopic dialog as the conversation in which the student asks questions and the expert answers with stories. They go on to propose a hypermedia system with which the student can interact in the expert's absence. The value of stories in architectural education, where every building is unique, is particularly significant (Heylighen et al. 2007, Fruchter and Cavallin 2006). CoMem builds on top of these efforts by aspiring to tell the story visually of how building designs evolved.

Storytelling was chosen as a metaphor in CoMem for two different but related reasons. Firstly, storytelling is how expertise is usually transferred in professional 
Demian, P. and Fruchter, R., 2009, "Effective visualisation of design versions: visual storytelling for design reuse" Research in Engineering Design, 19(4) 193-204.

design practice, and secondly, design rationale in CoMem is captured in the form of a story.

The first reason is more important because it relates directly to the user.

Storytelling is a useful metaphor because it matches how young designers interact with "human corporate memories", i.e. the experienced designers and mentors at design practices. Ethnographic observations (Demian and Fruchter 2006) of designers at work show that experienced designers tell stories. When instructing novice designers on how to reuse a component from a previous project, they tell stories about how this component was originally designed.

The second reason has to do with the nature of knowledge capture in the ProMem system, upon which CoMem is based. Knowledge capture in ProMem is processbased. Design rationale is captured as a history of the design process. Put simply, ProMem captures the story of how a team of designers got together and designed a building. It cannot be said to capture design expertise in any formal way such as by the formulation, application, or refinement of rules. But the design expertise possessed by the team members is manifest in the story of their collaboration. A detailed account of ProMem's approach for capturing design rationale is beyond the scope of this discussion (Fruchter et al. 1998). Briefly, Regli et al. (2000) contrast process-based approaches with feature-based approaches. Feature-based approaches capture design rationale as a series of logical moves within a precisely defined design space. Process-based approaches are useful when the problems are vague, there is little or no standardization of the designed artefact, and the design process is supported rather than automated. Feature-based approaches are useful for task specific contexts and narrow design domains where the domain knowledge can be formally encoded. Multidisciplinary building design falls into the former category. Almost no two buildings are the same, nor can the domain knowledge from the ten or so different disciplines that contribute to the design of a building be exhaustively codified. Building design problems are "wicked" (Rittel and Webber 1973). Instead, ProMem captures design rationale by supporting typical communication and coordination tasks that occur in building design teams. These include annotating the building model with notes, sharing data or documents linked to the building model, and sending change notifications to solicit feedback, give approval, broadcast changes, or initiate negotiations. Each time a change is detected (for example due to the addition of a note or a change in the CAD model), the system automatically creates a new version of the objects in question. This approach results in a relatively informal description of how the design evolved, but minimizes the additional effort required for knowledge capture. This is comparable to the approach by Conklin (1991) and others.

In spite of its informality, the ProMem design evolution history is extremely valuable. Schön (1983) notes that expertise (particularly in design) lies not in rules or plans entertained in the mind prior to action, but in the action itself. Before him, Polanyi (1966) coined the term tacit knowing to describe the fact that "we know more than we can tell" - that knowledge which shapes behaviour and yet is not ordinarily accessible to consciousness and so it is difficult to capture it directly. 
Demian, P. and Fruchter, R., 2009, "Effective visualisation of design versions: visual storytelling for design reuse" Research in Engineering Design, 19(4) 193-204.

More recently, researchers are beginning to recognize that design is a social process (Leifer 1997), and that design expertise lies not only in the individual designer's actions, but also in the interactions within a design team. Ferguson (1992, page 32) writes, "Those who observe the process of engineering design observe that it is not a totally formal affair, that drawings and specifications come into existence as a result of a social process. The various members of a design group can be expected to have divergent views of the most desirable way to accomplish the design they are working on... informal negotiations, discussions, laughter, gossip, and banter among members of a design group often have a leavening effect on the outcome." Arias et al. (1997) observe that "each stakeholder [in a design team] has a (sometimes narrow) view of the problem and an agenda to satisfy his/her particular goals. Stakeholders are often unaware that achieving their own goals can make things worse for other stakeholders." Bucciarelli (1994) concurs, proposing a model of the design process where each participant operates within a different "object world". Interestingly, Bucciarelli proposes "story making" as a useful metaphor for the process by which each participant understands the designed artefact.

If the design expertise cannot always be reduced to rules or procedures but is "in the design action" itself and much of what constitutes design action is the communication that goes on within a design team, then the story of how a design emerged from the communication within a design team can be said to capture to a large extent the designers' design rationale.

These two reasons - (1) design expertise is transferred in practice using stories, and (2) the design rationale is captured by ProMem in the form of a story - have in common their shared sense of a story as a conduit of knowledge transfer. What formal reasoning fails to grasp, a story simply conveys. The storytelling metaphor therefore allows the user to interact with a narrative of the evolution of a designed component. This narrative is useful in its own right, just as a story told by a mentor is useful.

\section{Visualizing Evolution Histories in CoMem}

Gershon and Page (2001) explore the link between storytelling and visualization. They propose two techniques: animation and frame-by-frame storytelling (the "comic book metaphor"). Garcia et al. (2002) use animation to communicate multidisciplinary design perspectives by adopting cinema storytelling techniques. Thomas and Calder (2001) note the value that cartoon animation techniques can add to graphical user interfaces.

CoMem Evolution History Explorer adopts the frame-by-frame technique, which gives the user more control over which parts of the story to explore and enables him/her to compare multiple versions side-by-side. The evolution history for a CoMem object has a tree structure. Consequently, the story is not linear but may involve several design alternatives being explored in parallel. CoMem retrieves the versions of any item and any attached content from the database and visualizes the version history using a node-link diagram, where each node is a version in the evolution history and a "frame" (or panel, to use comic book terminology) in the story. 
Demian, P. and Fruchter, R., 2009, "Effective visualisation of design versions: visual storytelling for design reuse" Research in Engineering Design, 19(4) 193-204.

The versions are laid out on a canvas (Figure2). Each version is represented as a colour-coded circle. The colour of the outline of the circle denotes its level of importance flag (low, conflict, or milestone), and the colour of the centre of the circle denotes its level of sharing flag (private, public, or consensus). This information is provided by the original design team working in ProMem. They are able to go back and flag various versions according to their level of importance and level of sharing. Any content linked to this version is also displayed as an icon linked to the circle. The user double-clicks on the icon to see a full view of the content. If the content is a piece of text (a note, change notification, or data) or an image, the full view is inserted into the canvas. For external documents that cannot be displayed on the canvas, double-clicking on the icon opens that document in an external window using the appropriate application.

The user is able to interact with this story in three ways. The user is able to:

1. Pan and zoom around the canvas.

2. Directly manipulate individual items on the canvas to move them or scale them.

3. Filter out versions based on their levels of importance or sharing.

Figure 2 to Figure 5 illustrate a typical series of interactions with the Evolution History Explorer. Figure 1 shows the initial form of the CoMem Evolution History Explorer. In Figure 2, the user filters out unimportant versions and chooses to see only important versions flagged as milestones by the original designers. The number of versions displayed falls from 15 to 8 . In Figure 3, the user zooms in on one version that appears to be the final version, with a lot of content attached. In Figure 4, the user shows the content attached to the desired version by double-clicking on the icons. Texts and images are displayed on the canvas; binary files are opened in the appropriate application in separate windows. In this case, there are photographs of the structure as built, which are displayed in the same window. There is also a handwritten note which is opened in an external Internet browser window.

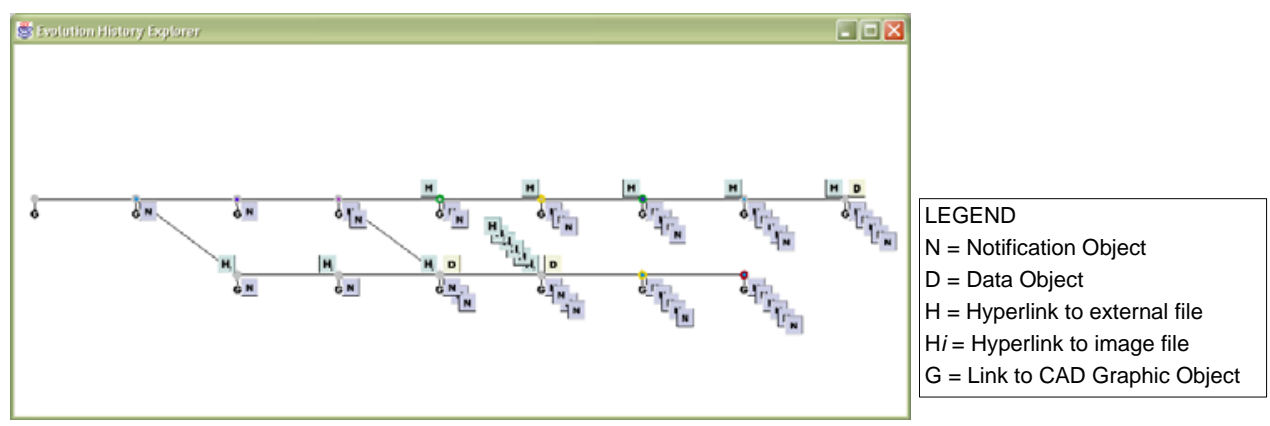

Figure 2: An example of the CoMem Evolution History Explorer for a particular item. 
Demian, P. and Fruchter, R., 2009, "Effective visualisation of design versions: visual storytelling for design reuse" Research in Engineering Design, 19(4) 193-204.

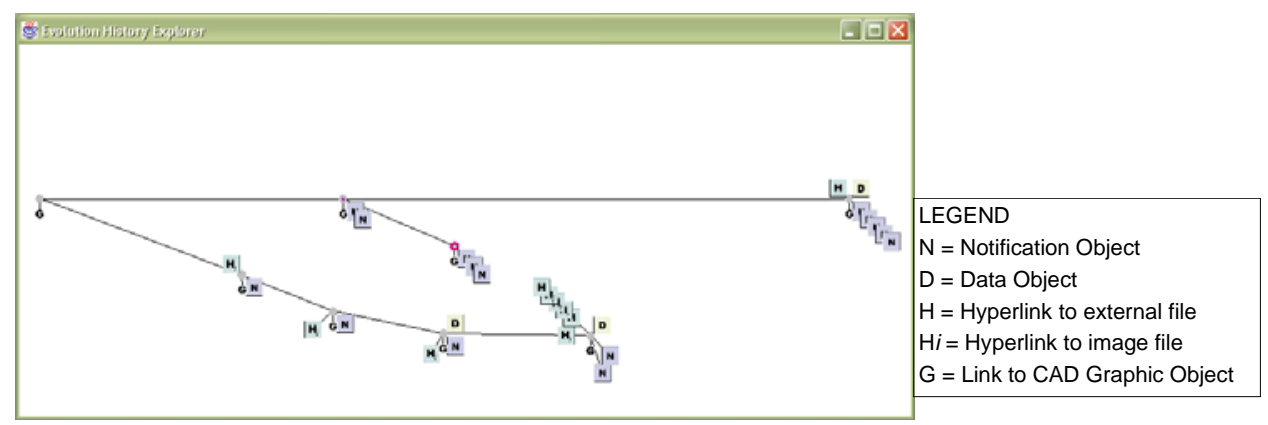

Figure 3: The user filters out unimportant versions.

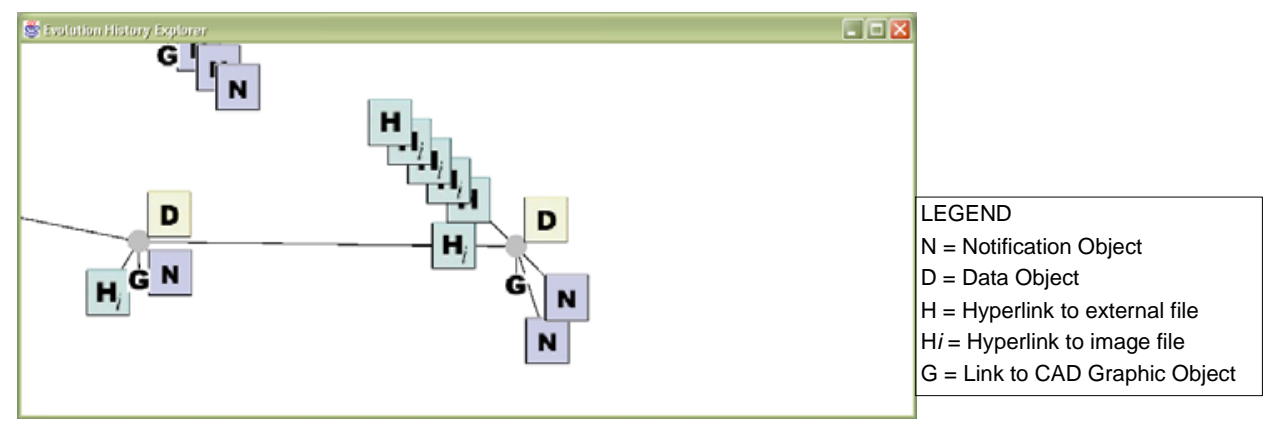

Figure 4: The user zooms in on one version.

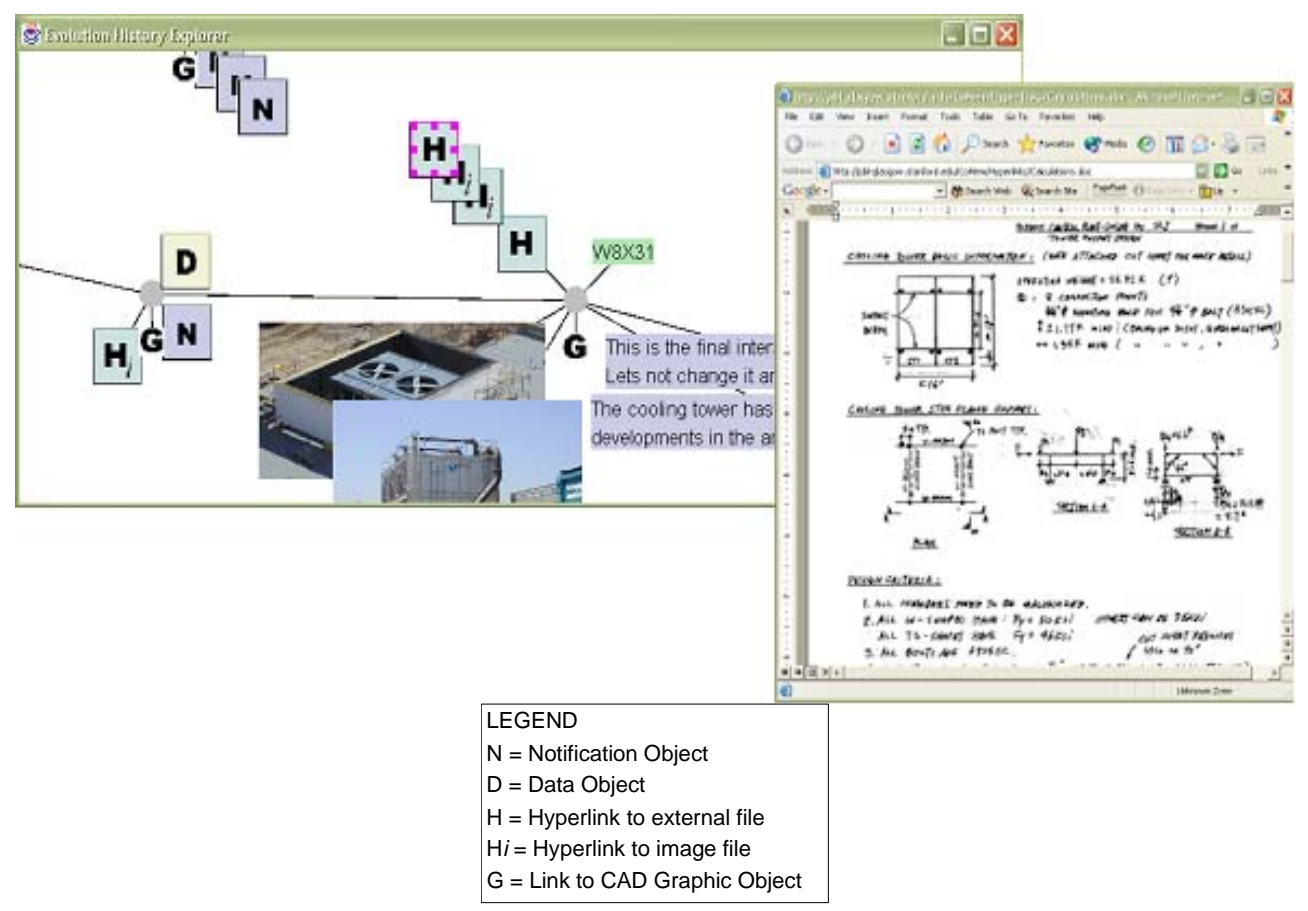

Figure 5: The user views the content attached to the desired version by double-clicking on the icons. Texts and images are displayed on the canvas; binary files are opened in the appropriate application in separate windows.

Like all CoMem modules, the Evolution History Explorer is Java-based and so can conceviably be adapted to run as an applet in a web browser or can be integrated with web-based PDM applicsations. 
Demian, P. and Fruchter, R., 2009, "Effective visualisation of design versions: visual storytelling for design reuse" Research in Engineering Design, 19(4) 193-204.

\section{Usability Evaluation of CoMem and Validation of the Storytelling Paradigm}

Usability evaluation of CoMem was conducted through formal user testing to determine the extent to which the visual storytelling paradigm supports the understanding of items found, and the extent to which this ability to understand improves the effectiveness of the reuse process. Detailed methodology and results of the CoMem evaluation are published elsewhere (Demian and Fruchter 2006). The important point to make here is that the results show that visual storytelling helps the user to understand the item considered for reuse. Since it is difficult to evaluate statements such as "designer can find and understand" or "external reuse is effective" in absolute terms, the strategy of the evaluation was to identify metrics for the validity of such statements and then to compare these metrics for CoMem versus "traditional tools". For the purpose of the evaluation, CoMem was compared to two other interfaces representing the state of practice in industry: Hit List, modelled after Internet search engines, and Outline Tree modelled after file and folder explorers in modern operating systems and PDM tools. User test subjects were asked to complete design reuse tasks using each of the three prototype systems.

A typical task was: "You are working on the design of a high-rise hotel structure. Find any content in the corporate memory that would be useful." The three prototype systems were plugged into the same underlying repository of content. Only the effectiveness of the user interfaces was assessed. The CoMem Evolution History Explorer, with its storytelling paradigm, was compared to text-based lists of versions in the other two interfaces, i.e., Hit List and Outline Tree. Figure 6 shows a typical series of interactions with the Hit List prototype where the user is searching for all items with the terms hotel and roof. The user chooses to see a listing of the versions for the Architecture discipline object (Figure 6 (b)). When a particular version is chosen, it is displayed in a new window (Figure 6 (c)). The version listing in the Outline Tree was similar.

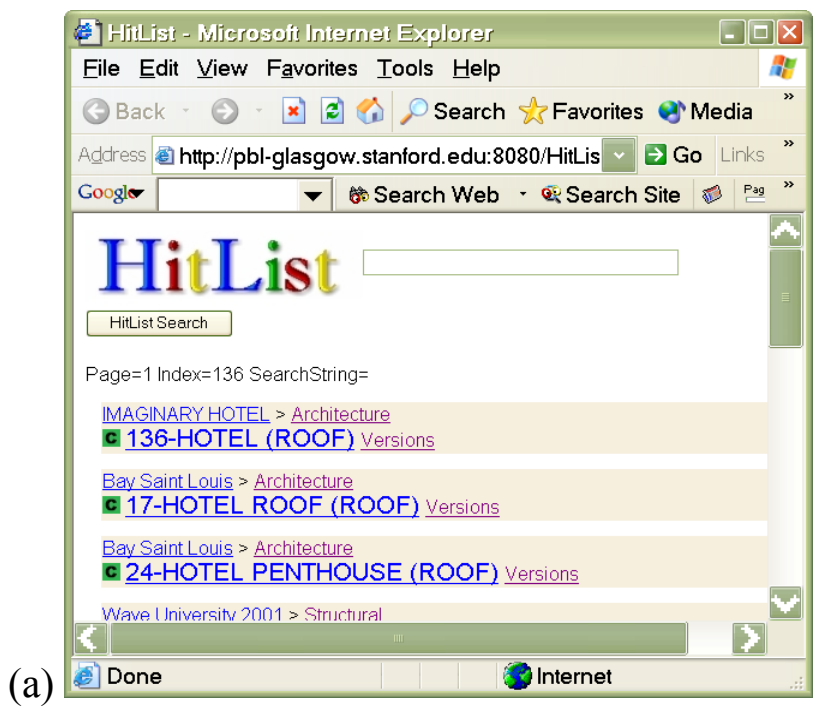


Demian, P. and Fruchter, R., 2009, "Effective visualisation of design versions: visual storytelling for design reuse" Research in Engineering Design, 19(4) 193-204.

(b)

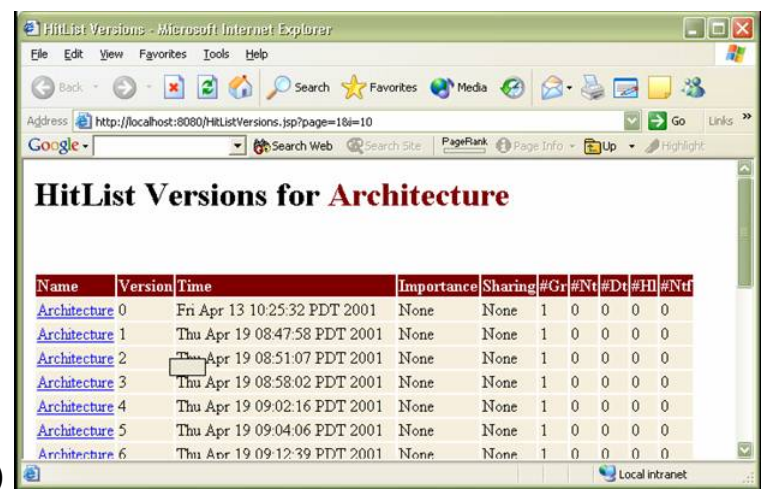

(c)

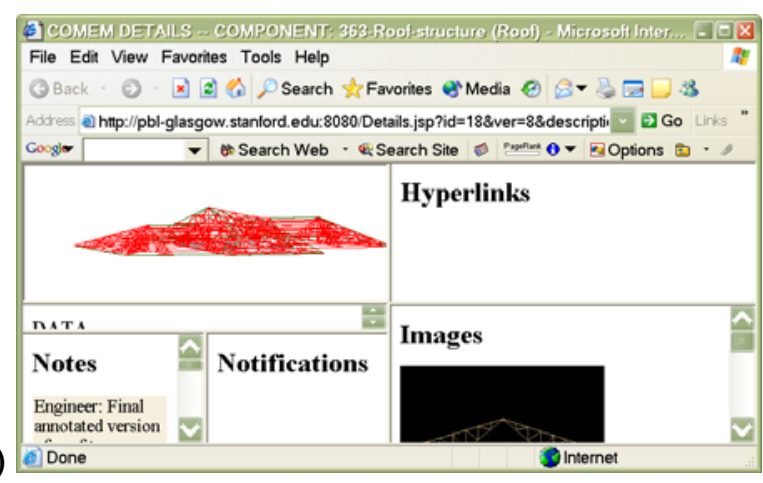

Figure 6: Hit List prototype. (a) Main page of Hit List for searching the corporate memory. (b) Web-based listing of versions of item selected from main page. (c) Web view of a version selected from the version list.

The following metric for effective understanding was measured:

- Ability to answer a set of questions after exploring the project context and evolution history, such as: "Why did the design team choose that building material?" A context score was calculated for each user by dividing the number of correctly answered questions by the total number of questions asked. This was intended to measure the extent to which the tool enabled the user to understand why that item was designed the way it was.

For effective external reuse, the extent to which the user agrees with the following statements was used as a measurable metric that assesses the effectiveness of the reuse process:

- If I had this system at work, I would reuse content from previous projects more frequently than I do currently.

- If I had this system at work, I would reuse content from previous projects more appropriately than I do currently.

For the purpose of the evaluation twenty participants were recruited from amongst students and researchers in the Department of Civil and Environmental Engineering at Stanford University, as well as professionals from local design offices. The participants were chosen to be as close as possible in age, computer experience and design experience to eliminate any variability in the data due to these factors. The evaluation sessions were conducted using a pilot corporate memory consisting of 10 project objects, 35 discipline objects, and 1036 components (before versioning). Of the 1036 component objects, approximately $30 \%$ were annotated with note objects at various points in their version histories. Each object in the pilot corporate memory had a version history spanning at least 5 versions. 
Demian, P. and Fruchter, R., 2009, "Effective visualisation of design versions: visual storytelling for design reuse" Research in Engineering Design, 19(4) 193-204.

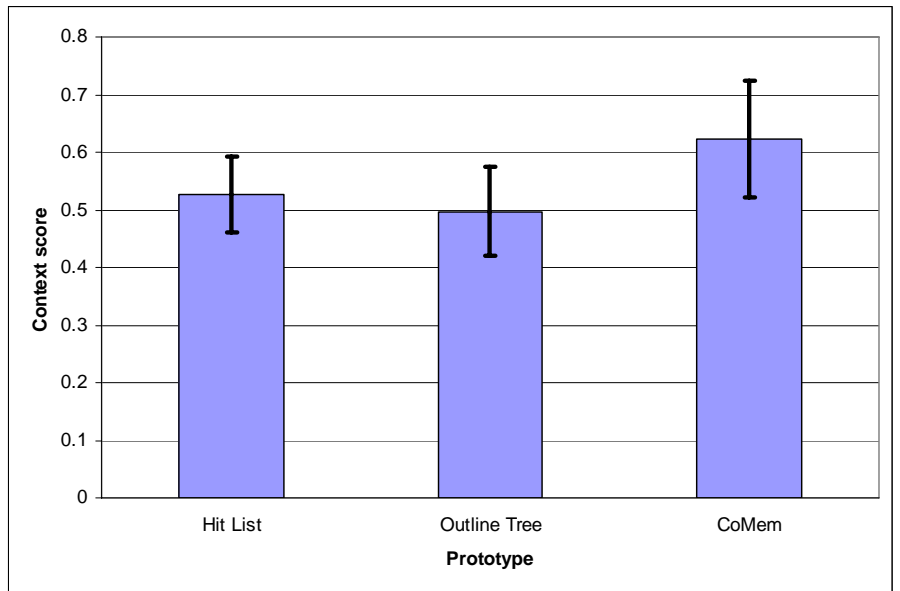

Figure 7: Context score during exploration tasks with $90 \%$ confidence intervals displayed.

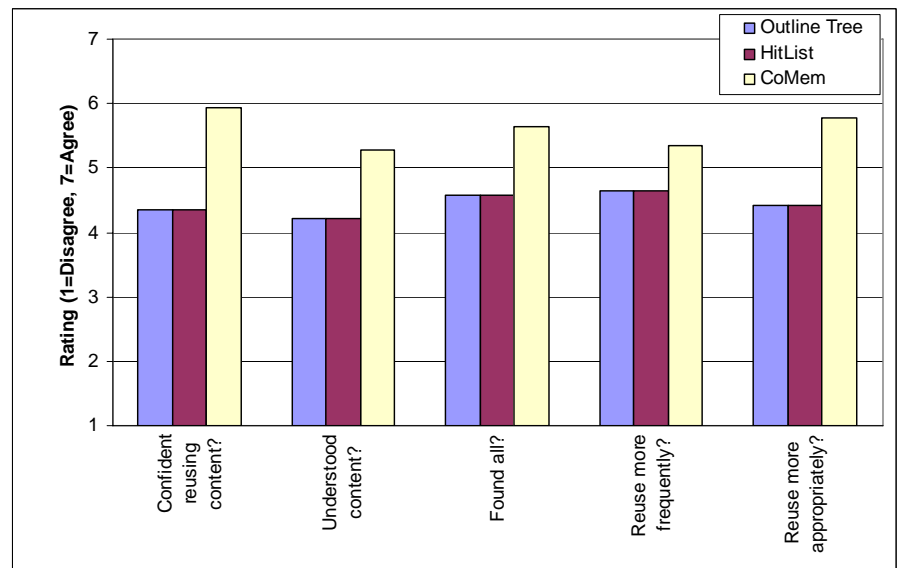

Figure 8: Examples of the questionnaire results.

Figure 7 shows the fraction of contextual questions that could be answered correctly by test participants about the items they retrieved. CoMem performed better than the Outline Tree and Hit List. It also had a slightly larger confidence interval. Most of the contextual questions were based on interactions between the designers, and the resulting version history of the item in question. The CoMem Evolution History Explorer was rated very highly by test participants.

Figure 8shows the subjective feedback of the test participants about CoMem, the Outline Tree, and Hit List, as gathered from questionnaires. For the questions regarding general usability characteristics (learnable, complicated, cumbersome), which are not displayed in Figure 8 for brevity, CoMem attained comparable scores to the Hit List and Outline Tree.

The first three questions in Figure 8 measure the user's perceived ability to find and understand:

- I would feel very confident reusing content that I found using this system.

- I had a good understanding of the items I was exploring.

- I felt that I was able to find all potentially reusable items in the corporate memory in the given time.

The higher scores attained by CoMem support the claim that storytelling is an effective paradigm for visualising version histories. 
Demian, P. and Fruchter, R., 2009, "Effective visualisation of design versions: visual storytelling for design reuse" Research in Engineering Design, 19(4) 193-204.

CoMem received higher scores particularly for the last two questionnaire questions in Figure 8, questions that were used as metrics to evaluate the extent to which external reuse is effective: does the user feel that if he/she had that prototype at work, they would reuse designs more frequently and more appropriately. Identical content was made available for reuse $\mathrm{n}$ all three tools; the only difference was the interface. The effectiveness of reuse using CoMem can be attributed in part to its storytelling paradigm for visualising version histories.

\section{Discussion: Why does visual storytelling work?}

Earlier in this paper, two objectives for the Evolution History Explorer were laid down. These are that it should enable the user (1) to view and reuse intermediate versions, and (2) to understand the particular version being reused and learn from the expertise of the original designers by seeing their rationale.

The first objective is met since CoMem Evolution History Explorer clearly allows the user to see intermediate versions of the design. The degree to which the second objective is supported depends on how much content the original designers attached to their shared product model. It is assumed, for the sake of discussion, that the corporate memory is fairly richly annotated such that:

- most of the annotations that designers would normally make on paper drawings are included in the database in the form of note and data objects; and

- most of the files and documents that would normally be exchanged amongst team members by fax or e-mail are included as hyperlink objects.

Given that this data is in place, how does the CoMem's visual storytelling help the user to understand the particular version being reused and gain valuable design expertise? The strength of the Evolution History Explorer is that it enables the user to see, interact with, and therefore compare multiple versions

simultaneously. The user can consider each version as an episode in a larger story rather than as an isolated event. These comparisons can be made longitudinally (i.e. forwards and backwards exploration of the evolution history, Figure 9) or sideways (i.e. sideways exploration of the evolution history, Figure 10). Such comparisons would not be possible if the versions were displayed in a list, or if the user could only see one version at time.

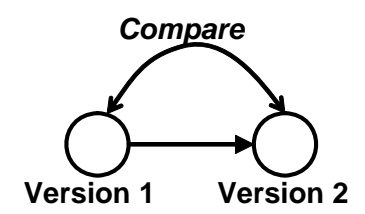

Figure 9: Longitudinal comparisons: comparing successive versions of any item from the corporate memory.

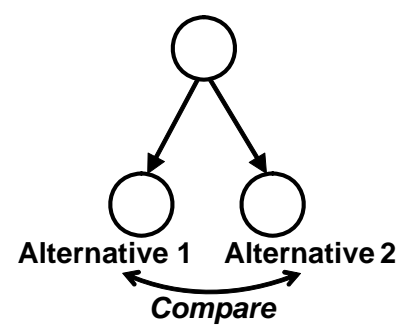

Figure 10: Sideways comparisons: comparing alternatives of any item from the corporate memory. 
Demian, P. and Fruchter, R., 2009, "Effective visualisation of design versions: visual storytelling for design reuse" Research in Engineering Design, 19(4) 193-204.

These comparisons are possible because the user is able to directly manipulate two or more versions and place them side-by-side. The user can then pan and zoom to obtain a good view of those versions, and expand some of the content attached to them and examine it. It is possible to view large amounts of content. Because of zooming, the space available is virtually unlimited. The combination of the three functions above - (1) moving and scaling individual items, (2) panning and zooming on the canvas, and (3) filtering items based on flags give the user the complete freedom to generate the desired view and make comparisons in order to understand the story.

ProMem captures versions automatically each time even the smallest change is made to the design. As a result, the number of versions can be very large, with insignificant changes between consecutive versions. This might not reflect the user's idea of a version. For this reason, the ability to filter by flags specified by the original design team is extremely useful. If the team flagged two versions as milestone versions, then these versions were probably meaningful milestones with important design developments occurring between them. The filters enable the user to make more meaningful comparisons by ignoring insignificant versions and preventing information overload (Figure 11). This filtering can either exclude intermediate versions in between milestone versions in the case of longitudinal comparisons (Figure ) or insignificant versions in two or more parallel design alternatives in the case of sideways comparisons.

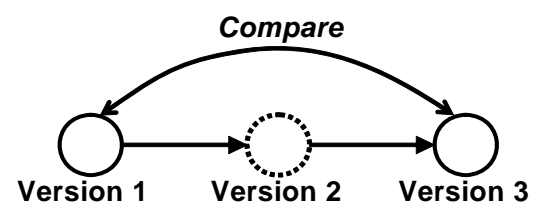

Figure 11: Comparisons across filtered versions.

As the original designers made decisions, their rationale is recorded in the archived content: the notes and notifications they exchanged and the information from external documents such as vendor catalogues or results from analysis and simulation programs. These act as snippets of rationale, with the user filling in the gaps as much as possible.

Emerging theories of comic book rhetoric (Duncan 1999, McCloud 1993, Eisner 1996) provide clues as to the effectiveness of this visual storytelling, particularly the concept of encapsulation: the framing of essential moments of a story in significant images. In the case of comic books, the creator makes the decision of what moments of the story to present. In CoMem, the user and the original designers jointly play this role. The original designers flag versions according to their levels of importance and sharing, and the user can filter according to these flags. Thus, the CoMem user and the original creators of the content collaboratively generate a coherent story our of the messy log of the design process.

Duncan (1999) notes that encapsulation is a reductive process, i.e. the creators reduce the story to moments on a page by encapsulation. Readers expand the isolated moments represented in discrete panels into a continuous story by closure, the process by which they "fill in the gaps". The placement of panels side-by-side is essential for this process of closure. 
Demian, P. and Fruchter, R., 2009, "Effective visualisation of design versions: visual storytelling for design reuse" Research in Engineering Design, 19(4) 193-204.

For example, a version of a hotel roof structure may be linked to a note from the engineering consultant explaining the complexity of designing structural members for the proposed architectural form of the roof. The next version of the roof may comprise a much simpler roof proposal. Seeing those two design versions sideby-side (annotated with exchanged text messages and images) makes it easier to understand the evolution of a design and the rationale driving this evolution.

\section{Conclusions}

The CoMem Evolution History Explorer visually lays out the versions from the evolution history onto a canvas, with each version linked to its parent (Figure 12). The user is able to filter out unimportant versions and modify the initial arrangement by translating and scaling the elements so that important versions are positioned close to each other. Seeing the versions side-by-side facilitates the comparison akin to the process of closure by which the reader of a comic book reconstructs a story from a series of discrete moments. Terry and Mynatt (2002) note the importance of seeing different versions simultaneously side-by-side, but in their case this is important to support rapid and fluid experimentation and explore alternatives during creative work.

Because the user is able to "see" the story, he/she will be able to explore and understand the rationale of the original designers in a way that would not be possible if the evolution history was presented as a flat list of versions or as a set of static images using a flipbook metaphor.

CoMem is still limited, though, in that can only display isolated evolution histories for individual objects. In reality, the evolution of a component object, for example, would be tightly linked to the evolution of its parent discipline object. The full story could only be told if both evolution histories were simultaneously displayed. Integration of multiple related evolution visualisations is a possible avenue for future research..

In conclusion, formal evaluation of CoMem supports the claim that visual exploration of design evolution helps in the understanding of previous designs and in effective design reuse. Storytelling is a helpful metaphor to be adopted in a tool to explore design evolution. A study of comic book theory provides clues as to the effectiveness of visual storytelling.

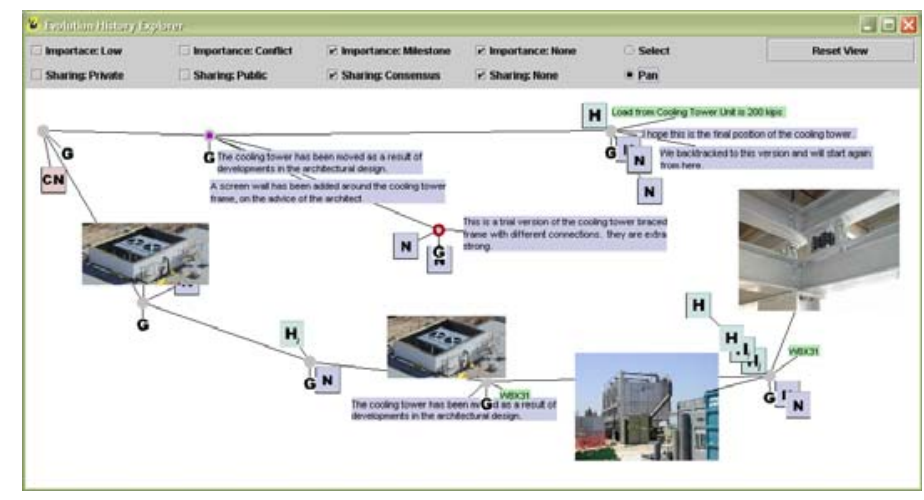

Figure 12: An example of the value of seeing different versions side-by-side in the CoMem Evolution History Explorer. 
Demian, P. and Fruchter, R., 2009, "Effective visualisation of design versions: visual storytelling for design reuse" Research in Engineering Design, 19(4) 193-204.

\section{Acknowledgements}

This research was funded by the UPS Endowment Fund, the Project-Based Learning Laboratory (PBL Lab) and the Center for Integrated Facility Engineering (CIFE) at Stanford University. The CoMem Evolution History Explorer uses the Java Jazz Library for zoomable interfaces by HCIL at the University of Maryland.

\section{References}

Aha DW, McSherry D, Yang Q, 2006. "Advances in conversational case-based reasoning." The Knowledge Engineering Review 20(3) 247-254.

Arias E., Eden H., and Fischer G., 1997. "Enhancing communication, facilitating shared understanding, and creating better artifacts by integrating physical and computational media for design", Symposium on Designing Interactive Systems (DIS), Proceedings of the Conference on Designing Interactive Systems: Processes, Practices, Methods, and Techniques.

Association of Swedish Engineering Industries 2001. "Product Data Management and Software Configuration Management - Similarities and Differences", online document indexed by CiteSeer and accessed on 31 August 2007: http://citeseer.ist.psu.edu/cache/papers/cs/25656/http:zSzzSzwww.mrtc.m dh.sezSzpublicationszSz0373.pdf/dahlqvist01 product.pdf

Ball L. J., Lambell N. J., Ormerod T. C., Slavin S., Mariani J. A. 2001. "Representing design rationale to support innovative design reuse: a minimalist approach", Automation in Construction, 10 (6), pp 663-674.

Barber J, Jacobson M, Penberthy L, Simpson R, Bhatta S, Goel A, Pearce M, Shankar M \& Stroulia E 1992. "Integrating Artificial Intelligence and Multimedia Technologies for Interface Design Advising." NCR Journal of Research and Development, 6(1):75-85, October 1992.

Bruls D. M., Huizing K., and van Wijk J. J., 1999. "Squarified treemaps”, Data Visualization 2000, Proceedings of the Second Joint Visualization Symposium organized by the Eurographics and the IEEE Computer Society Technical Committee on Visualization and Graphics (TCVG), (de Leeuw W. and van Liere R., Eds.), Springer-Verlag, Vienna, Austria, pages 33-42.

Bucciarelli L. L., 1994. Designing Engineers, MIT Press, Cambridge, MA.

Carroll J. M, Alpert S. R., Karat J., Deusen M. V., Rosson M. B., 1994. "Raison d'Etre: capturing design history and rationale in multimedia narratives", Conference on Human Factors in Computing Systems archive, Proceedings of the SIGCHI conference on Human factors in computing systems: celebrating interdependence .

Crnkovic I, Dahlkvist A, D. Svensson, 2001. “Complex Systems Development Requirements - PDM and SCM Integration” Second Asia-Pacific Conference on Quality Software (APAQS'01) p. 0427.

Conklin J, 1991. "A Process-Oriented Approach to Design Rationale." HumanComputer Interaction, 1991, 6(3\&4) 357-391.

Culley S. J., 1998. "Design reuse of standard parts", Proceedings of the Engineering Design Conference on Design Reuse, pages 77-88.

Davis J. E., (Ed.), 2002. Stories of Change: Narrative and Social Movements, State University of New York Press, Albany, NY. 
Demian, P. and Fruchter, R., 2009, "Effective visualisation of design versions: visual storytelling for design reuse" Research in Engineering Design, 19(4) 193-204.

Demian, P. and Fruchter, R., 2006, “ A Methodology for Usability Evaluation of Corporate Memory Design Reuse Systems.” ASCE Journal of Computing in Civil Engineering, November 2006.

Demian, P. and Fruchter, R., 2006, "An Ethnographic Study of Design Knowledge Reuse in the Architecture, Engineering and Construction Industry" Research in Engineering Design, volume 16, number 4, pp. 184-195.

Duncan R., 1999. "Toward a theory of comic book communication", Presented at the 85th Annual Convention of the National Communication Association (NCA). Also available from the Academic Forum Online, (Fudge K., Ed.), 1999-00, Number 17, Henderson State University, Arkadelphia, AR.

Eisner W., 1996. Graphic Storytelling. Poorhouse Press.

El-khoury J, 2005. "Model data management: towards a common solution for PDM/SCM systems" Proceedings of the 12th international workshop on Software configuration management, pp 17-32.

Ferguson E. S., 1992. Engineering and the Mind's Eye. MIT Press, Cambridge, MA.

Ferguson W, Bareiss R, Birnbaum L and Osgood R, 1992. “ASK Systems: An Approach to the Realization of Story-Based Teachers." Journal of the Learning Sciences 1992, 2(1) 95-134.

Finger S., 1998. "Design reuse and design research - Keynote paper", Proceedings of the Engineering Design Conference 1998: Design Reuse, (Sivaloganathan S. and Shahin T. M. M., Eds.), pages 3-10.

Fruchter R. and Demian P., 2002. "CoMem: Designing an interaction experience for reuse of rich contextual knowledge from a corporate memory", Artificial Intelligence for Engineering Design, Analysis and Manufacturing (AI EDAM), Volume 16, Issue 3, pages 127-147.

Fruchter R., 1996. "Conceptual, collaborative building design through shared graphics", IEEE Expert: Intelligent Systems, AI in Civil and Structural Engineering, Volume 11, Number 3, pages 33-41.

Fruchter R., Reiner K., Leifer L., and Toye G., 1998. "VisionManager: A computer environment for design evolution capture", Journal of Concurrent Engineering: Research and Applications (CERA), Volume 6, Number 1, pages 71-84.

Fruchter R and Cavallin HE, 2006. "Developing methods to understand discourse and workspace" AI \&Soc (2006)20:169-188.

Fulford R., 1999. The Triumph of Narrative: Storytelling in the Age of Mass Culture, House of Anansi Press, Toronto, Canada.

Garcia A. C. B., Carretti C. E., Ferraz I. N., and Bentes C., 2002. "Sharing design perspectives through storytelling", Artificial Intelligence for Engineering Design, Analysis and Manufacturing (AI EDAM), Volume 16, Issue 3, pages 229-241.

Gershon N. and Page W., 2001. "What storytelling can do for information visualization", Communications of the ACM, Volume 44, Number 8 , pages 31-37.

Harder WM, Ritter N \& Steiert HP, 2000. "Generating versioning facilities for a design-data repository supporting cooperative applications." International Journal of Cooperative Information Systems 9(1\&2) 117-146.

Heylighen A, Martin WM and Cavallin H, 2007. "Building Stories Revisited: Unlocking the Knowledge Capital of Architectural Practice" Architectural Engineering and Design Management 2007 (3) 65-74. 
Demian, P. and Fruchter, R., 2009, "Effective visualisation of design versions: visual storytelling for design reuse" Research in Engineering Design, 19(4) 193-204.

Jerding D. F. and Stasko J. T., 1994. "Using visualization to foster objectoriented program understanding", Georgia Institute of Technology, Atlanta, GA, Graphics, Visualization, and Usability Center (GVU), Technical Report GIT-GVU-94-33.

Kazman R. and Carrière S. J., 1998. "View extraction and view fusion in architectural understanding", Proceedings of the Fifth International Conference on Software Reuse (ICSR), pages 290-299.

Kolodner JL, Cox MT, Gonzalez-Calero P, 2006. "Case-based reasoning-inspired approaches to education." The Knowledge Engineering Review 20(3) 299-303.

Kuffner T. A. and Ullman D. G., 1990. "The information requests of mechanical design engineers", Proceedings of the Second International Conference on Design Theory and Methodology (DTM), ASME Design Engineering Technical Conferences (DETC), pages 167-174.

Leifer L., 1997. Design Project Laboratory (ME310), Stanford University, Course Notes.

Liu DT, Xu W, 2001. "A review of web-based product data management systems", Computers in Industry 44(3) 251-262.

Malmqvist J., 1995. “. "A Computer-Based Approach Towards Including Design History Information in Product Models and Function-Means Trees", Proceedings of DTM 1995.

McClatchey R, Kovacs Z, Estrella F, Le Goff J-M, Chevenier G, Baker N, Lieunard S, Murray S, Le Flour T, Bazan A 1998. "The integration of product data and workflow management systems in a large scale engineering database application." Database Engineering and Applications Symposium, 1998. Proceedings. IDEAS'98, pp296-302.

McCloud S., 1993. Understanding Comics: The Invisible Art, Kitchen Sink Press, Northampton, MA.

Polanyi M., 1966. The Tacit Dimension, Doubleday, Garden City, NY.

Popova M., Johansson P., and Lindgren H., 2002. "An integrated platform for case-based design", Proceedings of the CIB W78 Conference, Distributing Knowledge in Building, Volume 2, pages 99-106.

Regli W. C., Hu X., Atwood M., and Sun W., 2000. "A survey of design rationale systems: Approaches, representation, capture and retrieval", Engineering with Computers, Volume 16, Numbers 3-4, Springer-Verlag, Vienna, Austria, pages 209-235.

Retkowsky F., 1998. "Software reuse from an external memory: The cognitive issues of support tools", Proceedings of the Tenth Workshop on Psychology of Programming Interest Group (PPIG).

Rittel H W J, \& Webber M M, 1973. "Dilemmas in a general theory of planning." Policy Science, 4, 155-169.

Schank R. C., 1990. Tell Me a Story: A New Look at Real and Artificial Memory, Scribner, New York, NY.

Schön D. A., 1983. The Reflective Practitioner: How Professionals Think in Action, Basic Books, New York, NY.

Schonhoff jM, Dittrich KR, Strassler M, 2001. "Version Propagation in Federated Database Systems.” 2001 International Database Engineering \& Applications Symposium (IDEAS '01) p.0189.

Shneiderman B., 1999. "Supporting creativity with advanced informationabundant user interfaces", University of Maryland, College Park, MD, The Institute for Systems Research (ISR), Technical Report 1999-73. Also 
Demian, P. and Fruchter, R., 2009, "Effective visualisation of design versions: visual storytelling for design reuse" Research in Engineering Design, 19(4) 193-204.

available from Frontiers in Human-Centred Computing, Online Communities and Virtual Environment, 2001, (Earnshaw R., Guedj R., Van Dam A., and Vince J., Eds.), Springer-Verlag, London, UK, pages 469-480.

Simoff S. J. and Maher M. L., 1998. "Data mining in hypermedia case libraries", Proceedings of the Fifth International Conference on Artificial Intelligence in Design (AID), Machine Learning in Design (MLinD) Workshop.

Simmons A., 2001. The Story Factor: Secrets of Influence from the Art of Storytelling, Perseus Publishing, Cambridge, MA.

Terry M. and Mynatt E. D., 2002. "Supporting experimentation with side-views", Communications of the ACM, Volume 45, Issue 10, pages 106-108.

Thomas B. H. and Calder P., 2001. "Applying Cartoon Animation Techniques to Graphical User Interfaces", ACM Transactions on Computer-Human Interaction, Volume 8, Number 3, September 2001, pages 198-222.

Ye Y. and Fischer G., 2002. "Supporting reuse by delivering task-relevant and personalized information", Proceedings of the Twenty-Fourth International Conference on Software Engineering (ICSE), pages 513-523.

Zieliński K., Laurentowski A., Szymaszek J., and Uszok A., 1995. "A tool for monitoring heterogeneous distributed object applications", Proceedings of the Fifteenth International Conference on Distributed Computing Systems (ICDCS), pages 11-18. 